\title{
Anharmonicity and scissoring modes in the negative thermal expansion materials $\mathrm{ScF}_{3}$ and $\mathrm{CaZrF}_{6}$
}

\author{
T. A. Bird, ${ }^{1}$ J. Woodland-Scott, ${ }^{2}$ L. Hu, ${ }^{3}$ M. T. Wharmby $\odot,{ }^{4}$ J. Chen, ${ }^{3}$ A. L. Goodwin, ${ }^{2}$ and M. S. Senn $\circledast^{1, *}$ \\ ${ }^{1}$ Department of Chemistry, University of Warwick, Gibbet Hill, Coventry CV4 7AL, United Kingdom \\ ${ }^{2}$ Department of Chemistry, University of Oxford, Inorganic Chemistry Laboratory, South Parks Road, Oxford OX1 3QR, United Kingdom \\ ${ }^{3}$ Department of Physical Chemistry, University of Science and Technology Beijing, Beijing 100083, China \\ ${ }^{4}$ Deutsches Elektronen-Synchrotron (DESY), Notkestrasse 85, 22607 Hamburg, Germany
}

(Received 3 December 2019; accepted 4 February 2020; published 21 February 2020)

\begin{abstract}
We use a symmetry-motivated approach to analyzing $\mathrm{x}$-ray pair distribution functions to study the mechanism of negative thermal expansion in two $\mathrm{ReO}_{3}$-like compounds: $\mathrm{ScF}_{3}$ and $\mathrm{CaZrF}_{6}$. Both average and local structures suggest that it is the flexibility of $M$-F- $M$ linkages $(M=\mathrm{Ca}, \mathrm{Zr}, \mathrm{Sc})$ due to dynamic rigid and semirigid "scissoring" modes that facilitates the observed negative thermal expansion (NTE) behavior. The amplitudes of these dynamic distortions are greater for $\mathrm{CaZrF}_{6}$ than for $\mathrm{ScF}_{3}$, which corresponds well with the larger magnitude of the thermal expansion reported in the literature for the former. We show that this flexibility is enhanced in $\mathrm{CaZrF}_{6}$ due to the rocksalt ordering mixing the characters of two of these scissoring modes. Additionally, we show that in $\mathrm{ScF}_{3}$ anharmonic coupling between the modes responsible for the structural flexibility and the rigid unit modes contributes to the unusually high NTE behavior in this material.
\end{abstract}

DOI: 10.1103/PhysRevB.101.064306

\section{INTRODUCTION}

Research into materials that contract upon heating, termed negative thermal expansion (NTE) materials, has been steadily increasing over the past 30 years. The significance of the phenomenon was first underlined by Evans et al. in 1996 [1] by linking the large, isotropic NTE of $\mathrm{ZrW}_{2} \mathrm{O}_{8}$ to the crystal structure of the material, opening up the field to synthesis of new compounds. Since then, this field has been expanded to a wider range of materials, including simple oxides (such as $\mathrm{Cu}_{2} \mathrm{O}$ [2] and $\mathrm{ReO}_{3}[3,4]$ ) and metal-organic frameworks [5,6].

The rigid unit mode (RUM) model is a common way to explain the origin of NTE [7]. Materials made from rigid polyhedra have a significant energy barrier to distortions of the polyhedra, but a low barrier to collective dynamics such as rotations. These modes are often low in energy and so make a significant contribution to the coefficient of thermal expansion, and they can lead to NTE via the tension effect: if two linked bonds are straight or nearly straight and stretching the bonds would take a large amount of energy, a transverse displacement of the central atom would pull the two other atoms closer together, resulting in a local decrease in volume, the magnitude of which would increase when the temperature is raised [8]. $\mathrm{ReO}_{3}$, a material made from corner-sharing $\mathrm{ReO}_{6}$ octahedra (and hence can be thought of as an $A$-site-deficient perovskite), is commonly used to illustrate this model due

*m.senn@warwick.ac.uk

Published by the American Physical Society under the terms of the Creative Commons Attribution 4.0 International license. Further distribution of this work must maintain attribution to the author(s) and the published article's title, journal citation, and DOI. to the complexity of the motion in more typical NTE materials such as $\mathrm{ZrW}_{2} \mathrm{O}_{8}$. The octahedra in this material are expected to dynamically rotate in an out-of-phase manner with respect to their neighboring units about their average positions, resulting in a contraction of the structure while the material remains, on average, cubic [3]. Two compounds similar to $\mathrm{ReO}_{3}$ are studied herein: the isostructural $\mathrm{ScF}_{3}$ and the $A$-site-deficient double perovskite $\mathrm{CaZrF}_{6}$. Metal trifluorides adopting the $\mathrm{ReO}_{3}$ structure typically undergo a transition from the $P m \overline{3} m$ cubic structure to a rhombohedral phase $(R \overline{3} c)$ upon cooling, via long-range ordering of the $M \mathrm{~F}_{6}$ octahedra $\left(\mathrm{a}^{-} \mathrm{a}^{-} \mathrm{a}^{-}\right.$in Glazer notation). The dynamic motion of these tilts was expected to be the mechanism for NTE in $\mathrm{ScF}_{3}$ [9] supported by the fact that a phase transition to the rhombohedral tilt phase is observed under hydrostatic pressure of $0.7 \mathrm{GPa}$ at ambient temperature $[9,10]$ and in the related material $\mathrm{CoZrF}_{6}$, whose high-temperature phase is isostructural to $\mathrm{CaZrF}_{6}$ [11]. NTE is observed at a range of temperatures above the phase transition, but below it, once the phonon mode associated with the RUM has been "frozen in," strong positive thermal expansion is observed. Previous studies of these materials have shown large displacements of the fluoride ions perpendicular to the $M-\mathrm{F}-M$ bonds $(M=\mathrm{Sc}, \mathrm{Ca}, \mathrm{Zr})[12,13]$, consistent with a polyhedral rocking mechanism for NTE. Other studies have challenged the RUM model, concluding that only certain bonds were rigid $[14,15]$, rather than entire polyhedra, and that bond bending could be a contributor to NTE [16,17].

Several studies were performed recently to try and ascertain the origin of NTE in these materials. X-ray pair distribution function (PDF) analysis of two materials in the cubic $M \mathrm{ZrF}_{6}(M=\mathrm{Ca}, \mathrm{Ni})$ series has shown that differing degrees of flexibility in $M-\mathrm{F}$ linkages results in isostructural materials having very different thermal expansion properties [18]. Lattice dynamics calculations of $\mathrm{ScF}_{3}$ performed by Li et al. 
[19] showed mostly soft lattice modes that distorted the $\mathrm{ScF}_{6}$ octahedra; however, a $3 \times 3 \times 3$ grid of unit cells was chosen, which excludes the zone-boundary wave vectors which the RUMs are confined to. Molecular dynamics simulations on the general $\mathrm{ReO}_{3}$ structure [20], with variable interaction strengths, suggest a degree of flexibility in the octahedra enhances NTE. Another conclusion from these simulations was that a weaker anion-anion nearest-neighbor interaction enhances NTE, which is supported experimentally by the greater magnitude of NTE in $\mathrm{ScF}_{3}$ compared to $\mathrm{ReO}_{3}$. There is experimental evidence from Raman spectra and inelastic neutron scattering that the large NTE in these materials cannot be accurately predicted with the quasiharmonic approximation $[15,19]$, so subsequently lattice dynamics calculations were done to elucidate the connection between NTE and phonon anharmonicity since the relatively simple structure compared to other NTE materials allows for a more detailed analysis. These calculations show that cubic [21] and quartic [13,19] anharmonicity contribute significantly to the temperature dependence of the thermal expansion coefficient. Other simulations have shown that modes with quartic potential can have an enhanced NTE compared to a single-well potential [22].

$A B \mathrm{O}_{3}$ perovskites exhibit a wide range of octahedral tilt phase transitions, as classified by Glazer [23], yet do not generally display phonon-driven NTE. However, we have recently demonstrated how, by using a symmetry-motivated approach to analyzing PDF data, we can gain extra information on disorder and dynamics [24]. Our study on $\mathrm{BaTiO}_{3}$ showed that this method is very sensitive to soft phonon modes of RUMlike character. Here, we use this method to probe the character of the low-lying thermal excitations in the title compounds, where the amplitudes of such vibrations are believed to be very large.

\section{EXPERIMENTAL DETAILS AND DATA ANALYSIS}

Scandium trifluoride was used as supplied by Strem Chemicals. Synchrotron radiation $\mathrm{x}$-ray total scattering experiments were conducted at the synchrotron facility PETRA III (beamline P02.1 [25]) at DESY, Hamburg. A wavelength $\lambda=$ $0.2070 \AA$ was used to collect data. Data were collected at temperatures of $125,140,147$, and $152 \mathrm{~K}$ and at intervals of $25 \mathrm{~K}$ from 175 to $450 \mathrm{~K}$. The obtained two-dimensional images were masked and radially integrated using the DAWN [26] software. $G(r)$ and $D(r)$ functions were computed using GUDRUNX [27] using $Q_{\max }=21 \AA^{-1}$. GUDRUNX was also used to perform background subtraction and sample absorption corrections.

$\mathrm{CaZrF}_{6}$ was that prepared via a standard solid-state synthesis methods in Ref. [18]. The total scattering data were collected at 11-1D-C APS, Argonne National Laboratory, using a wavelength $\lambda=0.11798 \AA$ between 25 and $400 \mathrm{~K}$. The PDFs were computed using PDFGETX2 [28], which was also used for background subtraction and sample absorption corrections. $Q_{\max }=28 \AA^{-1}$ was used for the analysis presented below.

\section{A. Pair distribution function analysis}

Some form of modeling is usually required to extract information of interest, such as local distortions of atoms away from their high-symmetry positions, from pair distribution functions. The method presented here involves expanding the possible degrees of freedom in terms of symmetry-adapted displacements of the zone center and zone boundary irreducible representations (irreps) of the $P m \overline{3} m A$-site-deficient perovskite structure. For this analysis we use a parent $P m \overline{3} m$ perovskite with the $A$ site at the origin. Symmetry-breaking displacements transforming as the same irrep can be further decomposed into symmetry-adapted distortion modes by choosing a sensible basis that reflects the chemistry and crystallographic axes of the structure. The distortion modes have a 1:1 correspondence with phonon eigenvectors in the limit that only one set of atomic displacements transforms as the corresponding irrep. In cases where distortions from different Wyckoff sites transform as the same irrep, the character of the low-lying excitations can still be ascertained through refining the relative amplitudes of the individual distortion modes. An overview of the displacements that enter into each irrep was tabulated in a recent paper by Popuri et al. [29]. For both compounds, ISODISTORT [30] was used to generate a model parameterized in terms of symmetry-adapted displacements. A $2 \times 2 \times 2 \mathrm{P} 1$ supercell was used for $\mathrm{ScF}_{3}$ since this allows phonon modes with propagation vectors $k=\left[\begin{array}{lll}0 & 0 & 0\end{array}\right],[1 / 2$ 0 0], [1/2 1/2 0], and [1/2 1/2 1/2] to be modeled. While this is only a small fraction of possible wave vectors, these are both the ones that PDF data have the greatest sensitivity to and for which our symmetry-motivated approach provides the greatest number of constraints. Furthermore, even if the exact wave vectors of the NTE-driving phonons are of a longer wavelength, we still expect the character of those phonons to be reflected in our results, which probe a shorter wavelength. To generate the parametrization of $\mathrm{CaZrF}_{6}$, a $2 \times 2 \times 2$ supercell of disordered $\mathrm{Ca}_{0.5} \mathrm{Zr}_{0.5} \mathrm{~F}_{3}$ was used. The cations were then set to be fully ordered to generate the rocksalt ordered structure. In all refinements, the breathing mode about $\mathrm{Ca} / \mathrm{Zr}$ (transforming as $R_{2}^{-}$) was refined, making this description equivalent to the published $F m \overline{3} m$ structure [11]. The generated mode listings were output from ISODISTORT in CIF format and then converted to the INP format of the TOPAS ACADEMIC software, version 6 [31]. Modes transforming as the same irrep were tested simultaneously. An example of the best single-irrep refinement for each compound using this method is shown in Fig. 1. The results shown below (Fig. 2) were performed with a fitting range of $1\left(\mathrm{ScF}_{3}\right)$ or $1.7\left(\mathrm{CaZrF}_{6}\right)$ to $10 \AA$. The refinements were also done out to a higher radius; however, the results were broadly similar for these larger fitting ranges. A comparison between the results for 10 and $30 \AA$ can be seen in the Supplemental Material (Fig. S3) [32].

The thermal parameters for each site were modeled with a simple quadratic, i.e., $b_{i}=b_{i \text { low }}+u r+v r^{2}$, where $u$ and $v$ are constant across all sites for each refinement and $b_{i, \text { low }}$ is element dependent. While this does not capture the true physical behavior of the system, it was found to produce more robust fits to the data (stabler and fewer false minima) than other functional forms of $b_{i}$, with the results still being consistent with our analysis performed using different functional forms of $b_{i}$ (see Fig. S2 in the Supplemental Material).

To get an unbiased view of how each irrep influences the local structure, the refinement for each irrep was initiated from 

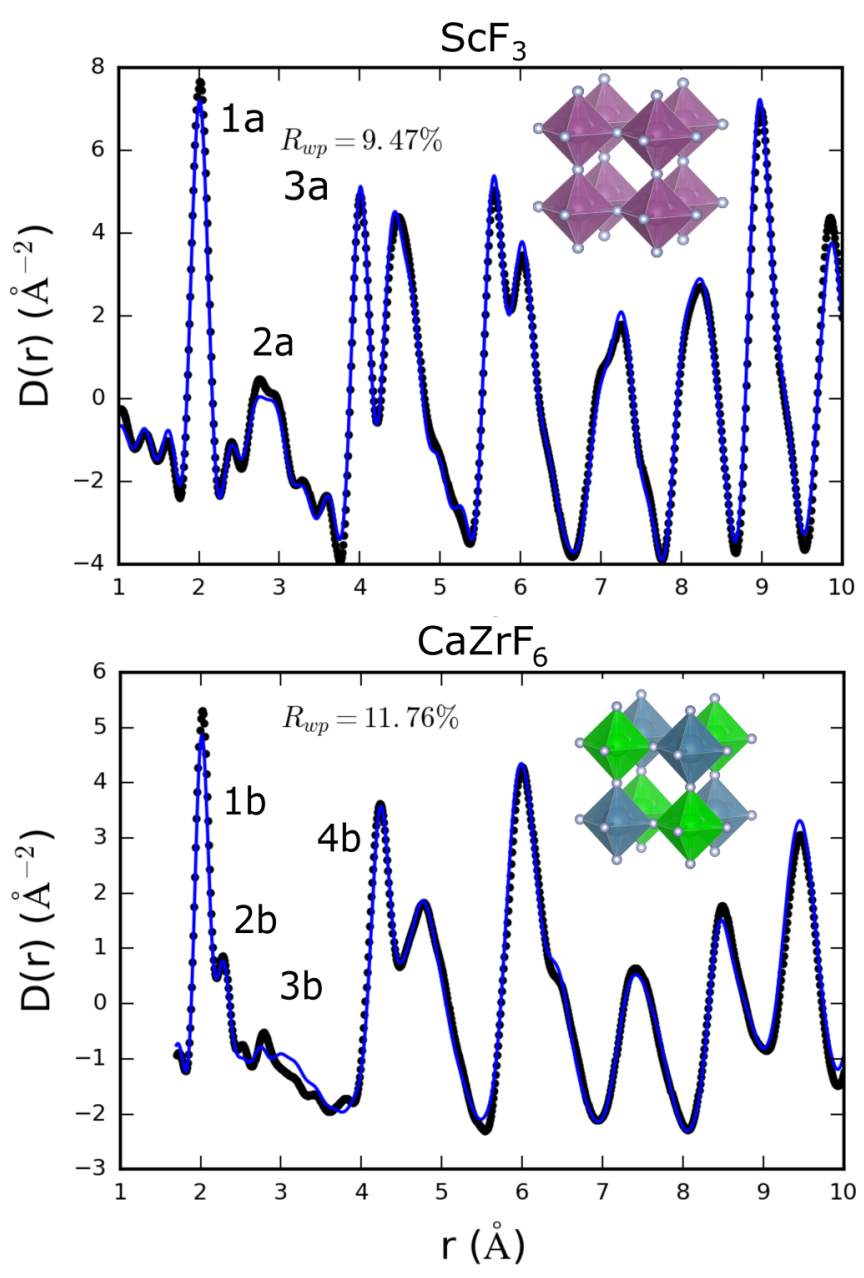

FIG. 1. Pair distribution functions for $\mathrm{ScF}_{3}$ (top) and $\mathrm{CaZrF}_{6}$ (bottom) at $400 \mathrm{~K}$ (black circles). A small box fit with the modes belonging to $X_{5}^{+}$refined is shown for both compounds (blue lines), with the $R_{2}^{-}$mode additionally refined for $\mathrm{CaZrF}_{6}$. Labeled peaks correspond to Sc-F (1a), F-F (2a, 3b), Sc-Sc (3a), Zr-F (1b), Ca-F (2b), and $\mathrm{Ca}-\mathrm{Zr}(4 \mathrm{~b})$.

randomized starting values of the relevant mode amplitudes. When a minimum was reached, the refined parameters were stored, rerandomized, and a new cycle was initiated. This process was repeated until 25000 iterations were reached (between 300 and 4000 refinements); this process was used to ensure a global minimum was reached for each mode. For refinements of atomic displacements transforming as the $\Gamma_{4}^{-}$irrep, corresponding to ferroelectric type distortions, the amplitudes of modes affecting the metal cations were used to fix the origin; otherwise, the mode amplitudes of this irrep would appear artificially high due to the floating origin of the unit cell. Finally, we note that if the refined mode amplitudes are treated as the mean absolute value of displacement of an harmonic oscillator, then the amplitude of the harmonic motion will be a factor of $\sqrt{2}$ larger than the refined values.

\section{B. Constrained order parameter directions}

Some order parameters can have many degrees of freedom associated with them. The exact number is a function of the degeneracy of the propagation vectors, the dimensionality of the irrep, and the number of distortions transforming as the irrep. All of these degrees of freedom are described by the collection of symmetry-adapted displacements or "distortion modes" that can be labeled accordingly. For example, in the parent structure $(P m \overline{3} m)$ of $\mathrm{ScF}_{3}$ there are three types of distortion that transform as $X_{5}^{+}$, which is two-dimensional and associated with the triply degenerate $k$ vector $\left[\begin{array}{lll}1 / 2 & 0 & 0\end{array}\right]$, which results in a total of 18 parameters, compared to just 3 for $M_{2}^{+}$(a triply degenerate single-dimensional $k$ vector) and $R_{5}^{-}$(a nondegenerate $k$ vector with three dimensions). In our refinements, to facilitate a fairer comparison between irreps, the order parameter direction (OPD) associated with the three wave vectors for each distortion have been set to the same values, i.e., the general OPD $(a, b ; c, d ; e, f)$ has been set to $(a, b ; a, b ; a, b)$. Different distortion modes of the same type associated with the $a$ and $b$ branches of the OPD are allowed to have different values. However, to further reduce the degrees of freedom that ratio between $a$ and $b$ across all distortion types that transform as a single irrep is fixed to be constant across different temperature ranges. This reduces the number of parameters for $X_{5}^{+}$from 18 to 4 . Physically, these approximations correspond to a harmonic approximation in which the order parameter directions with respect to the propagation vectors and irrep dimensionality are strictly degenerate in energy. An example of this implementation is given in the Supplemental Material.

\section{RESULTS AND DISCUSSION}

Rietveld refinement of $\mathrm{ScF}_{3}$ and $\mathrm{CaZrF}_{6}$ powder patterns can be used to gain some insight into the NTE behavior but can also be misleading; the average structure of both compounds remains cubic over the temperature ranges used; however, this structure fits the pair distribution function quite poorly, with PDFGUI [34] refinements of both structures from 1 to $10 \AA$ having $R_{w p} \approx 18 \%$ and $20 \%$ for $\mathrm{ScF}_{3}$ and $\mathrm{CaZrF}_{6}$, respectively (see Fig. S1 in the Supplemental Material). The average linear coefficient of thermal expansion $(\mathrm{CTE}) \approx$ $-7.5 \mathrm{ppm} \mathrm{K}^{-1}$ for $\mathrm{ScF}_{3}$ matches the literature reports well [9]. The measured $\mathrm{CaZrF}_{6}$ linear $\mathrm{CTE}$, as reported by $\mathrm{Hu}$ et al. from the same data [18], is $-6.69 \mathrm{ppm} \mathrm{K}^{-1}$. In the literature, $\mathrm{CaZrF}_{6}$ is reported to have a magnitude of NTE approximately two to three times that of $\mathrm{ScF}_{3}[9,11]$ for the temperature range $25-400 \mathrm{~K}$, whereas in these measurements they have quite similar values. The differences from literature reports are in part due to the differing temperature ranges over which CTEs are reported but may also be due to different strains, morphologies and thermal histories of samples [35,36]. The refined atomic displacement parameters (Fig. 2, top) reveal that most thermal motion of the $\mathrm{F}$ ions is perpendicular to the $M$-F- $M$ linkages $(M=\mathrm{Sc}, \mathrm{Ca}, \mathrm{Zr})$, indicating that a tensioning of these linkages could be responsible for the observed NTE.

Some information can be gained from the PDFs without any modeling. First, the effect of the rocksalt ordering of $\mathrm{Ca}^{2+}$ and $\mathrm{Zr}^{4+}$ in $\mathrm{CaZrF}_{6}$ can be seen in the presence of two peaks at $r \approx 2 \AA$, compared to just one in $\mathrm{ScF}_{3}$; the greater positive charge of $\mathrm{Zr}^{4+}$ compared to $\mathrm{Ca}^{2+}$ means the $\mathrm{F}^{-}$ions do not sit at the midpoint of Ca-F-Zr bonds (Fig. 1). Second, the relative magnitudes of the shorter interatomic separations (Sc-F, Sc-Sc; Ca-F, Zr-F, and Ca-Zr) means that the magnitude 


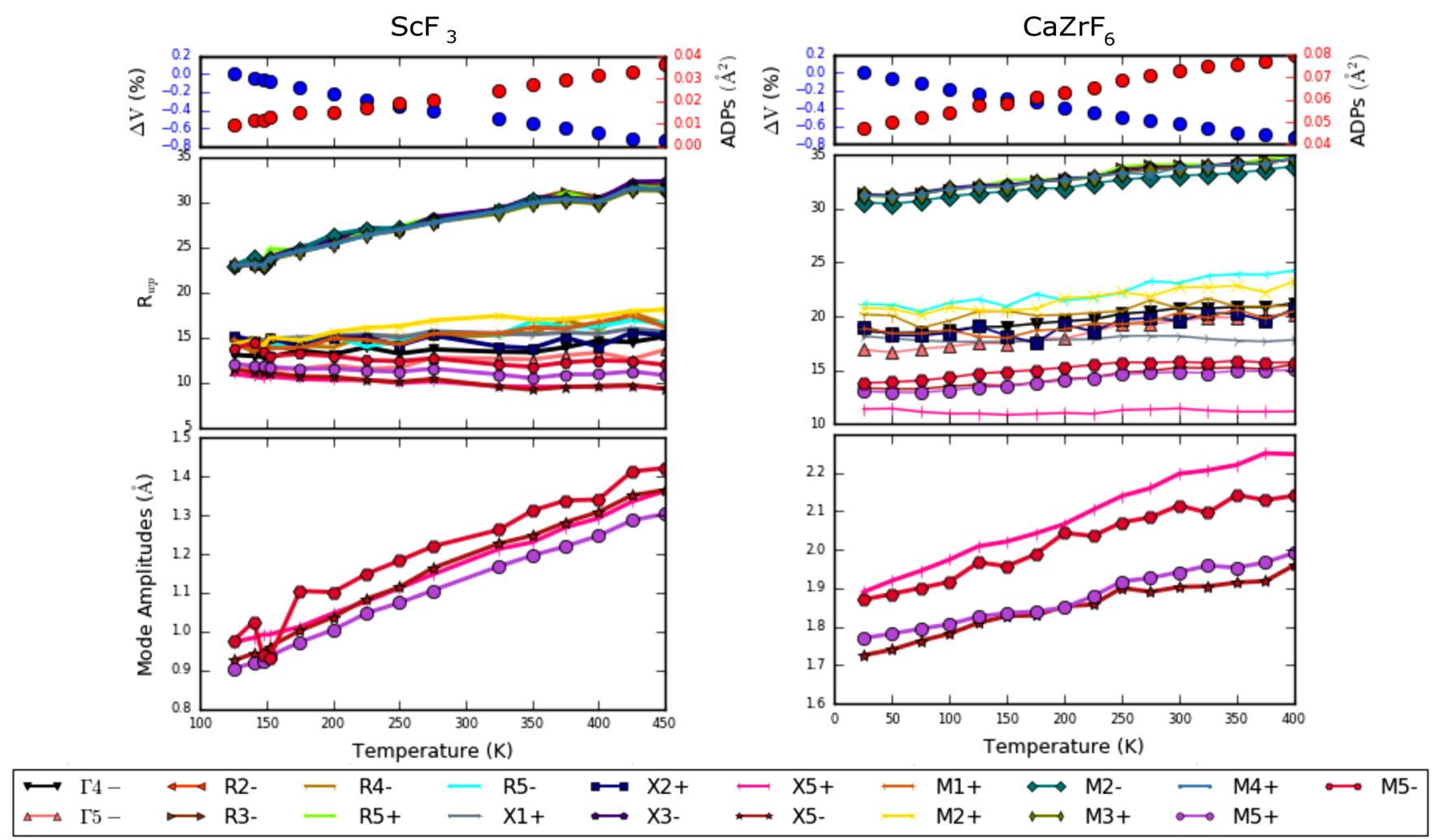

FIG. 2. Transverse atomic displacement parameters from Rietveld refinement (top), the best weighted-phase $R$ factor for each irrep at each temperature (middle), and the Boltzmann weighted mode amplitude (bottom). Results for $\mathrm{ScF}_{3}$ are displayed on the left; those for $\mathrm{CaZrF}_{6}$ are on the right.

of the mean $M$-F- $M$ angle ( $M=\mathrm{Sc}, \mathrm{Ca}, \mathrm{Zr}$ ) must deviate from $180^{\circ}$. The magnitude of this deviation is larger for $\mathrm{CaZrF}_{6}$ than for $\mathrm{ScF}_{3}$ (see Fig. S3). The first peak for $\mathrm{ScF}_{3}$ and the first two for $\mathrm{CaZrF}_{6}$ are noticeably less broad than the other peaks, indicating that the M-F bonds are relatively stiff. In contrast, the broadness of the F-F peaks at $\sim 3 \AA$ indicate a propensity for bending of the bond angles within the $\mathrm{MF}_{6}$ octahedra. Little further information can be gained from a simple inspection of the PDFs; hence, analysis of the structures has been performed in terms of symmetry-adapted displacements, as described in Sec. II A.

The results for the symmetry-adapted analysis are shown in Fig. 2 (middle and bottom). The distortions can be classed into three general types: rigid unit modes, consisting of coherent rotations of the octahedra; semirigid "scissoring" modes, where there is a scissoring of some of the M-F bonds within the octahedra; and bond-stretching modes, where some $M-\mathrm{F}$ bond lengths change. Most irreps in this analysis have only one distortion associated with them, although there are a few with more. There is a good degree of consistency between the two compounds; both have two "bands" of modes, one that fits well and one that fits poorly. The band with a greater weighted $R$ factor in both compounds consist of the same irreps $\left[R_{3}^{-}\right.$, $R_{5}^{+}, X_{3}^{-}, M_{2}^{-}, M_{3}^{+}, M_{4}^{+}$(and $R_{2}^{-}$in $\mathrm{ScF}_{3}$ )], all of which have distortions with a bond-stretching character. The rest of the irreps, in the band that fits the data well, have at least one distortion associated with them that has a rigid unit $\left(M_{2}^{+}\right.$and $R_{5}^{-}$) or scissoring mode character. There are four zone boundary irreps that consistently have the lowest weighted $R$ factor for both compounds for the majority of temperatures: $X_{5}^{+}, X_{5}^{-}$, $M_{5}^{+}$, and $M_{5}^{-}$. All of these irreps have one distortion associated with them that is of scissoring mode character. A depiction of the effect of these modes on the structure of $\mathrm{CaZrF}_{6}$ is shown in Fig. 3. The $\Gamma_{5}^{-}$irrep also fits well, especially in the refinements that go out to $30 \AA$. The displacements associated with this irrep are also of a scissoring mode character. However, despite the low $R_{w p}$, the mode amplitudes are consistently small; hence, most of the analysis is focused on $X_{5}^{+}, X_{5}^{-}, M_{5}^{+}$, and $M_{5}^{-}$. The weighted mean amplitudes over all refinements at each temperature for these irreps have been calculated and are shown in Fig. 2 (bottom), the weighting being given by a Boltzmann distribution, $w=\exp \left[\left(R_{\text {global }}-R_{w p}\right) / \sigma\right]$, where $R_{\text {global }}$ is the minimum weighted $R$ factor achieved across all refinements and all temperatures for the relevant compound and $\sigma$ is the value of a meaningful difference in the weighted $R$ factor, taken to be $0.1 \%$. $R_{\text {global }}$ is taken to be $9 \%$ for both compounds. The amplitudes of these modes $\left(X_{5}^{+}, X_{5}^{-}, M_{5}^{+}\right.$, and $M_{5}^{-}$) are consistently higher for $\mathrm{CaZrF}_{6}$ than for $\mathrm{ScF}_{3}$; this coincides well with the more significant distortion away from the average structure for $\mathrm{CaZrF}_{6}$, as seen in the mean $M$-F- $M$ bond angles and the greater magnitude of NTE reported in the literature. These modes also fit significantly better than the RUMs $\left(M_{2}^{+}\right.$and $\left.R_{5}^{-}\right)$. These best-fitting irreps $\left(X_{5}^{+}, X_{5}^{-}, M_{5}^{+}\right.$, and $\left.M_{5}^{-}\right)$are all two-dimensional and all have three $k$ vectors; therefore, the OPDs have been constrained as described in Sec. II B to allow for a fairer comparison with the RUMs, which have fewer degrees of freedom associated with them. For the $\mathrm{ScF}_{3}$, the unconstrained $R_{5}^{-}$(which is associated with 

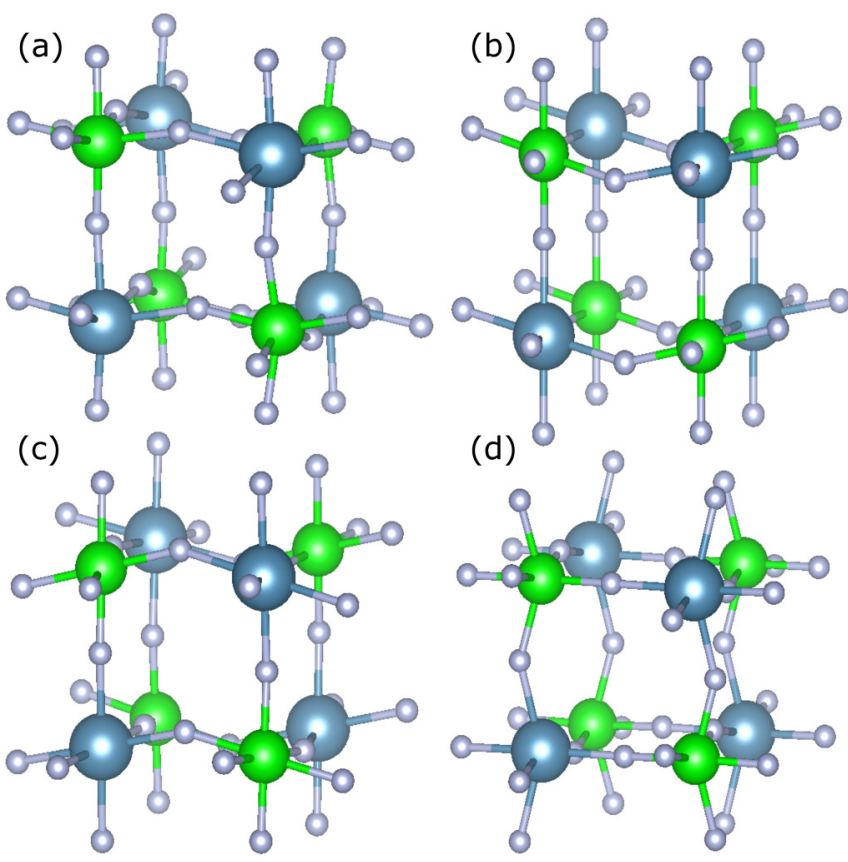

FIG. 3. Representations showing the effect of (a) $X_{5}^{+}$, (b) $X_{5}^{-}$, (c) $M_{5}^{+}$, and (d) $M_{5}^{-}$on the crystal structure of $\mathrm{CaZrF6}_{6}$. The distortions are taken from the refinements at $400 \mathrm{~K}$ with the lowest $R_{w p}$ and plotted using the VESTA software [33].

the out-of-phase octahedral tilts observed in other metal trifluorides) has a quality of fit similar to that of the constrained $X_{5}^{+}$, $X_{5}^{-}$, and $M_{5}^{+}$at lower temperatures and consistently performs better than $M_{5}^{-}$(Fig. 4). This suggests that a combination of both the rigid unit and scissoring modes is responsible for NTE, which agrees with a previous molecular dynamics study of these materials [20]. In that study the authors argued that correlated dynamics of flexible polyhedra result in a greater degree of NTE than purely rigid unit dynamics. However, for $\mathrm{CaZrF}_{6}$, we find the constrained scissoring modes, with the exception of $M_{5}^{-}$, consistently perform better than the RUMs. The RUMs also start to perform increasingly poorly as the temperature is raised above $100 \mathrm{~K}$, suggesting that the thermal expansion in $\mathrm{CaZrF}_{6}$ at higher temperatures may well be dominated by contributions from these scissoring modes. The increasing $R_{w p}$ of the RUMs as temperature is increased and the contrasting decrease in $R_{w p}$ seen for the scissoring modes tally well with the phonon dispersion curves of both compounds $[13,19]$. These show that the scissoring modes are slightly higher in energy than the RUMs, so the scissoring modes will become more active at higher temperatures.

As discussed earlier, the different charges on the two cations in $\mathrm{CaZrF}_{6}$ result in a need to refine the octahedral breathing mode, transforming as the $R_{2}^{-}$irrep, alongside the other distortion modes in order to facilitate a more direct comparison to $\mathrm{ScF}_{3}$. In the average structure of $\mathrm{CaZrF}_{6}$, this breathing mode is frozen in, lowering the symmetry from $P m \overline{3} m$ to $F m \overline{3} m$. This also has the effect of mixing the characters of some of the irreps such that the associated atomic displacements now transform as the same irrep. For example, the $X_{5}^{+}$and $M_{5}^{-}$irreps of $P m \overline{3} m$ correspond to the $X_{5}^{-}$irrep of $F m \overline{3} m$, and $X_{5}^{-}$and $M_{5}^{+}$correspond to $X_{5}^{+}$. To de-
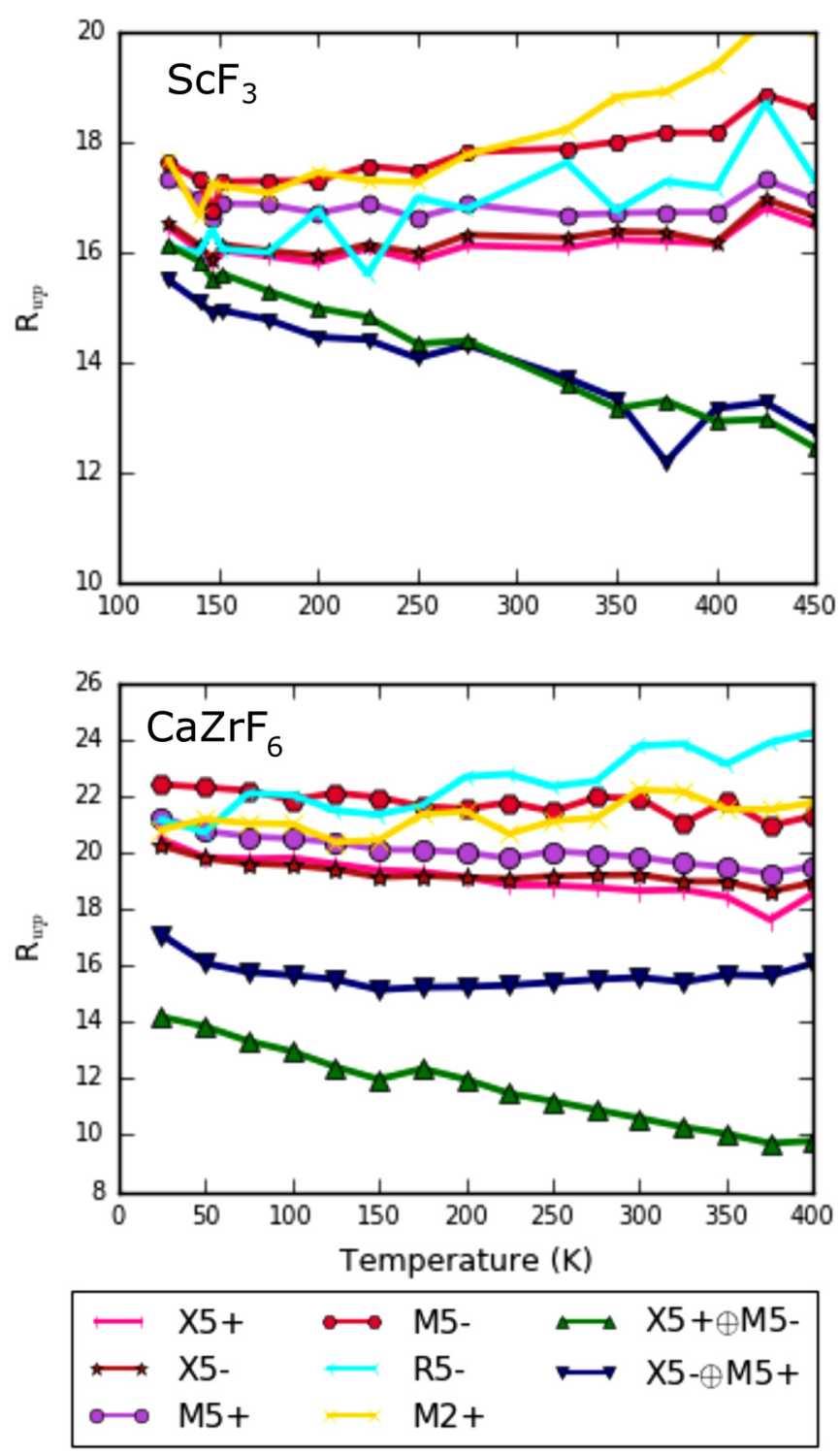

FIG. 4. Comparison of weighted $R$ factors for restricted irreps $X_{5}^{+}, X_{5}^{-}, M_{5}^{+}$, and $M_{5}^{-}$, unrestricted irreps $M_{2}^{+}$and $R_{5}^{-}$, and coupled $X_{5}^{+} \oplus M_{5}^{-}$and $X_{5}^{-} \oplus M_{5}^{+}$.

termine whether this mixing of characters has any effect on the observed local structure of $\mathrm{CaZrF}_{6}$, the constrained OPD $X_{5}^{+}$ and $M_{5}^{-}$modes were refined together (hereafter referred to as $X_{5}^{+} \oplus M_{5}^{-}$). This gave a significant improvement to the quality of the fit (Figs. 4 and 5). To determine whether this coupling is a significant effect, results are compared to a two-phase model, in which modes transforming as different irreps are refined in separate phases (Fig. 6). Hereafter these two models will be referred to as the "coupled" model (denoted with $\oplus$ ) and the "two-phase" model (denoted with \&). The coupled modes have a significantly better $R$ factor above $100 \mathrm{~K}$ but fit worse than the two-phase refinement below this temperature. The same comparisons are also done for $\mathrm{ScF}_{3}$, where any coupling between phonons of these characters should arise from only anharmonic interactions. In contrast to $\mathrm{CaZrF}_{6}$, which shows a clear preference for coupling between $X_{5}^{+}$and $M_{5}^{-}$, no evidence of such coupling and hence an anharmonic 

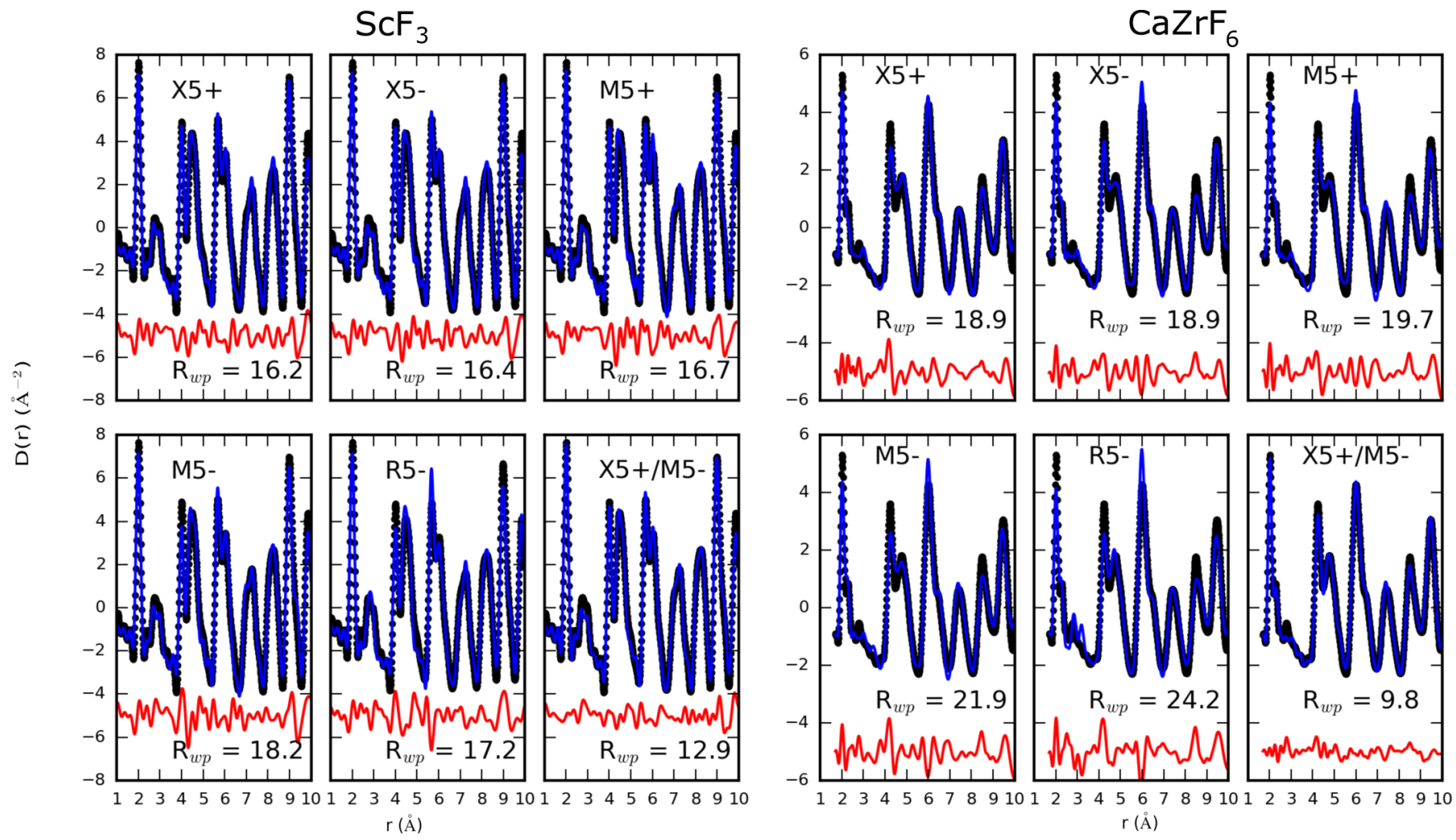

FIG. 5. Comparison of fits to $\mathrm{ScF}_{3}$ (left) and $\mathrm{CaZrF}_{6}$ (right) $\mathrm{PDF}$ data at $400 \mathrm{~K}$ using restricted $X_{5}^{+}, X_{5}^{-}, M_{5}^{+}$, and $M_{5}^{-}$; unrestricted $R_{5}^{-}$; and restricted $X_{5}^{+} \oplus M_{5}^{-}$.

interaction is seen here for $\mathrm{ScF}_{3}$. This suggests that while these scissoring modes are important in determining the local structure of $\mathrm{ScF}_{3}$, any anharmonic coupling between them has little influence on the lattice dynamics that drive NTE. A similar comparison is made for $X_{5}^{-} / M_{5}^{+}$; however, the two-phase refinements consistently fit better than the coupled model for both compounds. This may be due to both distortions locally having the same character (Eu of point group $m \overline{3} m$ ) with respect to the $\mathrm{MF}_{6}$ octahedra, making coupling unfavorable.

Next, we investigate if the similar quality of fits of the scissoring modes $X_{5}^{+}, X_{5}^{-}$, and $M_{5}^{+}$and the rigid unit mode $R_{5}^{-}$could be indicative that the two types of distortion are cooperatively coupled to produce the observed NTE. To test this hypothesis, we explore two scenarios: whether this observation is simply due to the dynamic distortions occurring in different sample volumes or at different times from each other or a coupled model which implies that significant anharmonic coupling between these modes is occurring. For both materials, the $X_{5}^{+} / R_{5}^{-}$refinements show a sort of behavior similar to the $X_{5}^{+} \oplus \mathrm{M}_{5}^{-}$refinements in $\mathrm{CaZrF}_{6}$, in that the refinements of the coupled modes perform worse than the two-phase refinements at lower temperatures but soon cross over to show an improved fit, although the results for $\mathrm{CaZrF}_{6}$ are not robust. Since by the symmetry lowering of the rocksalt ordering in $\mathrm{CaZrF}_{6} X_{5}^{+}$and $M_{5}^{-}$are allowed to couple and we have shown our analysis to be sensitive to this coupling, the results in Fig. 7 are indicative that there is coupling between the $X_{5}^{+}$and $R_{5}^{-}$modes. However, as, by symmetry, coupling in $X_{5}^{+} \oplus R_{5}^{-}$is not permitted on its own, we construct a coupled distortion that forms an invariant in the free-energy expansion by inclusion of the $M_{5}^{-}$irrep. The $X_{5}^{+}$and $M_{5}^{-}$
OPDs in this refinement are still restricted, resulting in three more parameters than the $X_{5}^{+} \oplus M_{5}^{-}$refinements but much improved fits (Figs. 4 and 7). This model results in a very good agreement with the data (Fig. 7).

A very recent analysis of $\mathrm{ScF}_{3}$ neutron PDF data using the reverse Monte Carlo (RMC) method by Dove et al. [37] similarly concluded that it is a combination of structural flexibility and RUMs that causes the NTE in the compound. Dove et al. argued that the flexibility of the structure allows RUMs and RUM-like modes to occupy a larger volume in reciprocal space, meaning they give a greater contribution to the overall thermal expansion behavior, compared to entirely rigid structures. Our results here echo this conclusion and underline the dominant contribution of scissoring modes in describing the fluctuations from the average symmetry. Additionally, in the work of Dove et al., geometric algebra was used to quantify the proportion of the motion of the atoms in $\mathrm{ScF}_{3}$ originating from correlated whole-body octahedral motion, deformations of the F-Sc-F right angles, and changes in the Sc-F bond length. This analysis resulted in a ratio of approximately $7: 2: 1$ of bends:rotations:stretches. The $X_{5}^{+} \& R_{5}^{-}$and $X_{5}^{-} \& R_{5}^{-}$twophase refinements described previously give a similar ratio of bends:rotations, approximately $8: 2$, although the contribution from stretches is negligible ( $<1 \%$ of the total motion). The $X_{5}^{+} \& R_{5}^{-}$refinements for $\mathrm{CaZrF}_{6}$ give an approximately $7: 3$ ratio of bends:rotations, again with a negligible contribution from stretches. There is hence a high degree of consistency between results derived via big-box RMC methods and those of our symmetry-motivated approach here. A different analysis of neutron PDF data of $\mathrm{ScF}_{3}$, performed by Wendt et al. [38], models the $\mathrm{F}$ atoms as being randomly positioned on a 

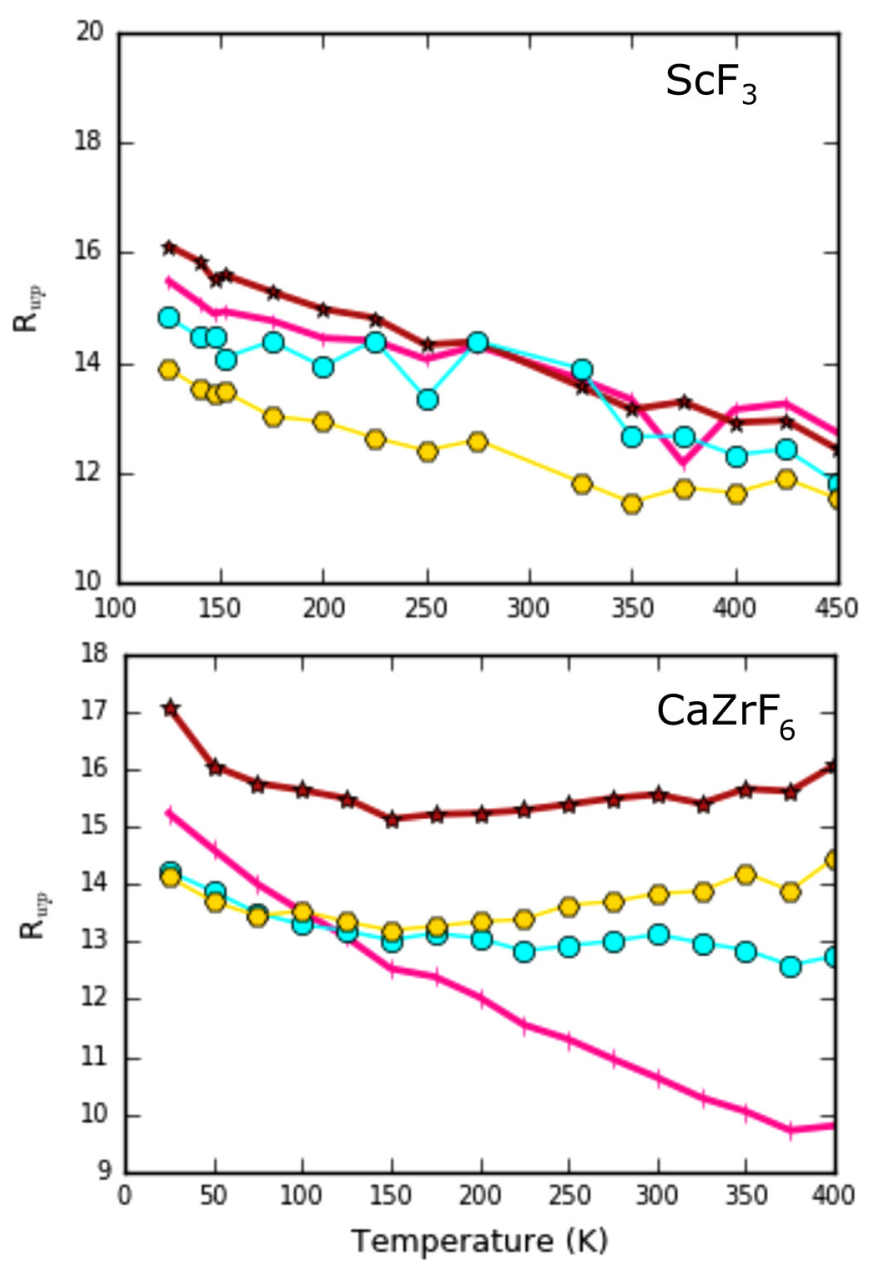

$\circ \quad \circ 55+\&$ M5- 2 phase $\circ \circ \mathrm{X5}-\&$ M5+ 2 phase

$\longmapsto X 5+\oplus$ M5- coupled $\quad \star X 5-\oplus M 5+$ coupled

FIG. 6. Comparison of coupled and two-phase fits to PDF data as a function of temperature for $\mathrm{ScF}_{3}$ (top) and $\mathrm{CaZrF}_{6}$ (bottom), as described in the text.

torus-shaped Gaussian distribution around the $\mathrm{F}$ sites in the average structure, with no correlation between neighboring $\mathrm{F}$ atoms, in a fashion similar to entropic elasticity in polymers. The model reproduces the observed NTE behavior and F-F distribution up to $\approx 700 \mathrm{~K}$. It shows how important the flexibility of Sc-F-Sc linkages is in this material, a fact consistent with our findings here; however, it fails to account for the full range of NTE in the material. The previously discussed RMC model shows that at least a small fraction of the motion of $\mathrm{F}$ atoms in the material can be accounted for by correlated rigid-unit-type distortions, results which are compatible with our symmetry-based analysis of the x-ray PDF data.

In summary, we have shown via a symmetry-motivated real-space analysis of PDF data that the most significant distortions in these $\mathrm{ReO}_{3}$-like NTE materials are scissoring modes, which involve scissoring of the $M \mathrm{~F}_{6}$ octahedral bond

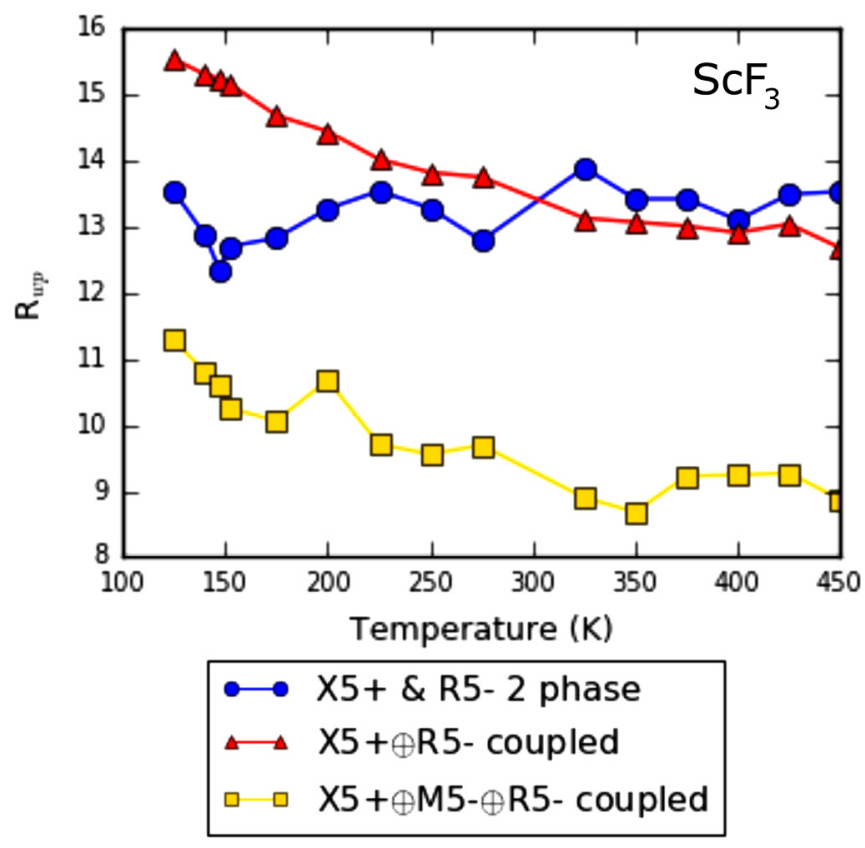

FIG. 7. Comparison of fits for $X_{5}^{+} \oplus R_{5}^{-}$using a two-phase model (blue) and a coupled model (red) and $X_{5}^{+}(a, b ; 0,0 ; 0,0) \oplus$ $M_{5}^{-}(0,0 ; c, d ; 0,0) \oplus R_{5}^{-}(e, f, g)$ (yellow) for $\mathrm{ScF}_{3}$.

angles. These modes have a greater amplitude in $\mathrm{CaZrF}_{6}$ than $\mathrm{ScF}_{3}$, which corresponds well to the greater magnitude of NTE reported in the literature for the former. Coupling between these modes and the rigid unit modes has been shown to be active and the likely origin of unusually high NTE in these structures.

The $\mathrm{ScF}_{3}$ data for this study are available as a supporting data set [39].

\section{ACKNOWLEDGMENTS}

T.A.B. thanks EPSRC for a Ph.D. studentship through the EPSRC Centre for Doctoral Training in Molecular Analytical Science, Grant No. EP/L015307/1. M.S.S. acknowledges the Royal Commission for the Exhibition of 1851 and the Royal Society for fellowships. We acknowledge DESY (Hamburg, Germany), a member of the Helmholtz Association HGF, for the provision of experimental facilities. Parts of this research were carried out at PETRA III. This research used resources of the Advanced Photon Source, a U.S. Department of Energy (DOE) Office of Science User Facility operated for the DOE Office of Science by Argonne National Laboratory under Contract No. DE-AC02-06CH11357. We acknowledge the measurement of PDF data by Dr. Y. Ren. Samples were characterized using the I11 beamline at the Diamond Light Source before total scattering experiments were performed. Access to this beamline was granted via Block Allocation Group EE18786.
[1] J. S. Evans, T. A. Mary, T. Vogt, M. A. Subramanian, and A. W. Sleight, Chem. Mater. 8, 2809 (1996).
[2] M. Dapiaggi, W. Tiano, G. Artioli, A. Sanson, and P. Fornasini, Nucl. Instrum. Methods Phys. Res., Sect. B 200, 231 (2003). 
[3] T. Chatterji, P. F. Henry, R. Mittal, and S. L. Chaplot, Phys. Rev. B 78, 134105 (2008).

[4] T. Chatterji, T. C. Hansen, M. Brunelli, and P. F. Henry, Appl. Phys. Lett. 94, 241902 (2009).

[5] I. Grobler, V. J. Smith, P. M. Bhatt, S. A. Herbert, and L. J. Barbour, J. Am. Chem. Soc. 135, 6411 (2013).

[6] N. Lock, Y. Wu, M. Christensen, L. J. Cameron, V. K. Peterson, A. J. Bridgeman, C. J. Kepert, and B. B. Iversen, J. Phys. Chem. C 114, 16181 (2010).

[7] M. T. Dove and H. Fang, Rep. Prog. Phys. 79, 066503 (2016).

[8] G. D. Barrera, J. A. O. Bruno, T. H. K. Barron, and N. L. Allan, J. Phys.: Condens. Matter 17, R217 (2005).

[9] B. K. Greve, K. L. Martin, P. L. Lee, P. J. Chupas, K. W. Chapman, and A. P. Wilkinson, J. Am. Chem. Soc. 132, 15496 (2010).

[10] S. U. Handunkanda, E. B. Curry, V. Voronov, A. H. Said, G. G. Guzmán-Verri, R. T. Brierley, P. B. Littlewood, and J. N. Hancock, Phys. Rev. B 92, 134101 (2015).

[11] J. C. Hancock, K. W. Chapman, G. J. Halder, C. R. Morelock, B. S. Kaplan, L. C. Gallington, A. Bongiorno, C. Han, S. Zhou, and A. P. Wilkinson, Chem. Mater. 27, 3912 (2015).

[12] L. Hu, J. Chen, A. Sanson, H. Wu, C. Guglieri Rodriguez, L. Olivi, Y. Ren, L. Fan, J. Deng, and X. Xing, J. Am. Chem. Soc. 138, 8320 (2016).

[13] M. K. Gupta, B. Singh, R. Mittal, and S. L. Chaplot, Phys. Rev. B 98, 014301 (2018).

[14] M. G. Tucker, A. L. Goodwin, M. T. Dove, D. A. Keen, S. A. Wells, and J. S. O. Evans, Phys. Rev. Lett. 95, 255501 (2005).

[15] A. Sanson, M. Giarola, G. Mariotto, L. Hu, J. Chen, and X. Xing, Mater. Chem. Phys. 180, 213 (2016).

[16] N. Sennova, R. Bubnova, J. Shepelev, S. Filatov, and O. Yakovleva, J. Alloys Compd. 428, 290 (2007).

[17] A. Senyshyn, B. Schwarz, T. Lorenz, V. T. Adamiv, Y. V. Burak, J. Banys, R. Grigalaitis, L. Vasylechko, H. Ehrenberg, and H. Fuess, J. Appl. Phys. 108, 093524 (2010).

[18] L. Hu, J. Chen, J. Xu, N. Wang, F. Han, Y. Ren, Z. Pan, Y. Rong, R. Huang, J. Deng, L. Li, and X. Xing, J. Am. Chem. Soc. 138, 14530 (2016).

[19] C. W. Li, X. Tang, J. A. Muñoz, J. B. Keith, S. J. Tracy, D. L. Abernathy, and B. Fultz, Phys. Rev. Lett. 107, 195504 (2011).

[20] J. T. Schick and A. M. Rappe, Phys. Rev. B 93, 214304 (2016).

[21] Y. Oba, T. Tadano, R. Akashi, and S. Tsuneyuki, Phys. Rev. Mater. 3, 033601 (2019).

[22] A. Sanson, Mater. Res. Lett. 7, 412 (2018).
[23] A. M. Glazer, Acta Crystallogr., Sect. B 28, 3384 (1972).

[24] M. S. Senn, D. A. Keen, T. C. A. Lucas, J. A. Hriljac, and A. L. Goodwin, Phys. Rev. Lett. 116, 207602 (2016).

[25] A.-C. Dippel, H.-P. Liermann, J. T. Delitz, P. Walter, H. SchulteSchrepping, O. H. Seeck, and H. Franz, J. Synchrotron Radiat. 22, 675 (2015).

[26] M. Basham, J. Filik, M. T. Wharmby, P. C. Chang, B. El Kassaby, M. Gerring, J. Aishima, K. Levik, B. C. Pulford, I. Sikharulidze, D. Sneddon, M. Webber, S. S. Dhesi, F. Maccherozzi, O. Svensson, S. Brockhauser, G. Náray, and A. W. Ashton, J. Synchrotron Radiat. 22, 853 (2015).

[27] S. E. McLain, D. T. Bowron, A. C. Hannon, and A. K. Soper, GUDRUN, a computer program developed for analysis of neutron diffraction data, Chilton, ISIS Facility, Rutherford Appleton Laboratory, 2012.

[28] X. Qiu, J. W. Thompson, and S. J. Billinge, J. Appl. Crystallogr. 37, 678 (2004).

[29] S. R. Popuri, R. Decourt, J. A. McNulty, M. Pollet, A. D. Fortes, F. D. Morrison, M. S. Senn, and J. W. Bos, J. Phys. Chem. C 123, 5198 (2019).

[30] B. J. Campbell, H. T. Stokes, D. E. Tanner, and D. M. Hatch, J. Appl. Crystallogr. 39, 607 (2006).

[31] A. A. Coelho, P. A. Chater, and A. Kern, J. Appl. Crystallogr. 48, 869 (2015).

[32] See Supplemental Material at http://link.aps.org/supplemental/ 10.1103/PhysRevB.101.064306 for Rietveld refinements, average structure refinements of PDF data and comparisons of different peak width functions.

[33] K. Momma and F. Izumi, J. Appl. Crystallogr. 44, 1272 (2011).

[34] C. L. Farrow, P. Juhas, J. W. Liu, D. Bryndin, E. S. Boin, J. Bloch, T. Proffen, and S. J. Billinge, J. Phys.: Condens. Matter 19, 335219 (2007).

[35] C. Yang, P. Tong, J. C. Lin, X. G. Guo, K. Zhang, M. Wang, Y. Wu, S. Lin, P. C. Huang, W. Xu, W. H. Song, and Y. P. Sun, Appl. Phys. Lett. 109, 023110 (2016).

[36] L. P. Prisco, C. P. Romao, F. Rizzo, M. A. White, and B. A. Marinkovic, J. Mater. Sci. 48, 2986 (2013).

[37] M. T. Dove, J. Du, Z. Wei, D. A. Keen, M. G. Tucker, and A. E. Phillips, arXiv:1905.09250.

[38] D. Wendt, E. Bozin, J. Neuefeind, K. Page, W. Ku, L. Wang, B. Fultz, A. Tkachenko, and I. Zaliznyak, Sci. Adv. 5, eaay2748 (2019).

[39] https://doi.org/10.6084/m9.figshare.11605278.v1. 